\title{
Food Supply Chain Safety Risk Prevention and Control: Based on the Behavioral Perspective*
}

\author{
Yong-Sheng Liu ${ }^{1}$, Rong $\mathrm{Yu}^{2}$, Xiang-Xiang Lin $^{2}$ \\ ${ }^{1}$ School of Business, Beijing Wuzi University, Beijing, China; ${ }^{2}$ Department of Graduate Students, Beijing Wuzi University, Beijing, \\ China. \\ Email: bjwylys@sina.com,yurongnick@sina.com
}

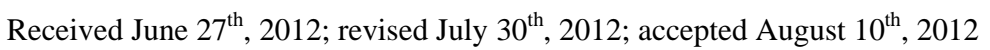

\begin{abstract}
Food supply chain is characterized by the complexity of covering a large range of aspects, which determines the difficulty of its safety risk prevention and control. Every step in food supply chain involves human activities, therefore the human participation becomes one of the critical elements that impact the food supply chain safety risk. From the perspective of human behavior as a cut-in point, the matter of food supply chain safety risk prevention and control is discussed and analyzed, referring to related theories of organizational behavior. Based on the discussion on attitude and motivation, two main factors that influence human behavior, approaches of controlling human behavior are summarized from two aspects, which are respectively correcting the food supply chain participants' attitudes and providing them with incentives, so as to reach the goal of food supply chain safety risk prevention and control.
\end{abstract}

Keywords: Food Supply Chain; Risk Prevention and Control; Behavior

\section{Introduction}

Food supply chain is characterized with its complexity, which is one of the most important reasons for what makes the matter of food safety complicated. There are a great deal of links in food supply chain, interconnected with each other, and as long as one of them is out of work, the problems of food safety will be aroused, even putting threats to human welfare. Therefore, food supply chain safety is critical to guarantee safe and efficient food supply. Unsafe elements of food supply exist in various places in food supply chain. They can be found in supplying link, in processing link, in transporting link, or in consuming link and the like [1]. These ubiquitous underlying problems usually owe to human behaviors. Human beings are indispensible in food supply chain, for the food supply chain is run only by different kinds of human activities. However, due to the limitation of perception, human do not always behave right, which leads to difference between behaviors and expectations, and generates food safety problems. Every participant in food supply chain behaves variously, even one person behaves differently in different period of time. This diversity of

\footnotetext{
*This work is supported by Project Supported by Academic Human Resources Development in Institutions of Higher Learning under the Jurisdiction of Beijing Municipality (PHR (IHLB)) under Grant PHR2012; Research on Business Operation and Business Service Innovative, Beijing Wuzi University (2012).
}

human behavior adds uncertainty to supply chain. Thus, human behavior is one of critical factors that determine the food supply chain safety risk, and preventing and controlling food supply chain safety risk from a behavioral perspective is becoming a practical problem that is urgent to be solved.

\section{Overview of Research on Food Supply Chain Safety Risk Based on Behavioral Perspective}

Food supply chain is an integrated system that guarantees a series of links organically and economically operate, ranging from raw material, producing and processing, restoring to sales and consuming. Every link in food supply chain involves human resources, so the researches on human behavior in supply chain refer to all links. As for researches in supply link of food supply chain, R. C. Cui et al. (2012) [2] analyzed the reasons of farmers' being shortsighted and utilitarian tendency, and also the effects of uncompleted contracts and supervision barrier on farmers' implicit breach of contract. Z. G. Wang et al. (2011) [3] analyzed key elements that affect farmers' decisions and behaviors on using pesticide and chemicals. In the researches in end consuming link, C. Z. Qiu et al. (2008) [4] demonstrated that safe food consumption behavior is one of key solutions to food safety problems, and put that food information symmetry is an important influencing 
factor to safe food consumption. L. Jiang (2011) [5] analyzed public perception to food safety risk, during which the influence of human behavior on risk perception was discussed from the view of both individual and group. C. M. Fan et al. (2012) [6] presented a relation model of risk perception and reaction, based on the empirical research on Sanlu milk powder incident. As for food supply chain surveillance link, Z. Y. Zhang et al. (2009) [7] undertook a game analysis between governmental supervision and food producing industry, demonstrating that the effectiveness of governmental supervision is determined by strengthening enforcement of punishment. As for researches in other links, P. D. Liu et al. (2008) [8] discussed the influence of personnel on the food supply chain safety risk. Through presenting a model for suggesting moral policy establishment in food supply chain, L. Manning et al. (2006) [9] analyzed a series of elements included in moral behavior in food supply chain. B. Bigliardi et al. (2010) [10] presented a performance measurement system, which can motivate managers to carry out much better operation plans to add corporate values. T. Tokar et al. (2010) [11] put that behavioral research can improve the quality of logistics and supply chain management theoretically and practically.

Concluded from above discussion, although human behavior has been referred in researches on food supply chain safety risk, the mechanism of behavior shaping has not been discussed yet. Taken influencing factors of behavior shaping as a cut-in point, with connecting human behavior and food supply chain safety risk, and through discussing the mechanism of behavior shaping, the behavior triggering food supply chain safety risk is analyzed essentially. The food supply chain safety risk prevention and control is discussed from the perspective of behavior control.

\section{Behavior and Food Supply Chain Safety Risk}

To a large extent, the food supply chain safety risk attributes to human factors. Human, as the important participant in the food chain, is responsible for the safety problems that occur in it. Human participates in all kinds of activities and is responsible for all the production. Even though the human workers are replaced by machines, the projects that they operate are made by human. Therefore, most of the safety problems should be attributed to human factors. Due to the subjective initiative and limited rationality [12], human behaviors cannot always accurately follow the instructions, but be more or less twisted by some personal reasons.

Behavior, as a psychological term, contains four aspects, which are respectively values, attitudes, perception and learning [13]. Attitude is an important one, for its impacting factors are easier to be found and get controlled. It is a psychological tendency toward the object which is set up on the basis of one's value or value system. Attitude is one of the most essential factors to behaviors. Different individual may hold different attitude on the same thing, the same activity or the same person, so they act in different ways [14]. Attitude is not innate, but formed gradually during the interaction with other people, groups and environment. There are several factors that influence the forming of attitude: the need, the knowledge, the group cognition, the individual identity and the individual experience. See as Figure 1.

Attitudes influence the direction of behaviors but it is not the only influence. The practice of a behavior needs incentives. Incentive process is a psychological activity that intrinsic motivations which makes people strive to their goal. The incentive process is started in an external stimulus which emerges from the incompatibility of individual and the surrounding environment caused by some kind of imbalance or insecurity, then the emotion begins to get nervous, and this tension is a performance of need. The need is another influence of attitude. When the external incentives can meet the needs, then the motive will occur and behavior will follow. When the behavior meets the need, then tension will be weakened. Thus the whole process of incentive will be complete. See as Figure 2.

This shows that human behavior plays a very important role in food chain safety control and an entry point. Those behaviors that cause risks are considered as "unsafe behaviors". To avoid unsafe behaviors, the attitudeforming factors and incentive factors should be changed to control the direction of attitude and the intensity of incentive, so that the behaviors of food chain participants

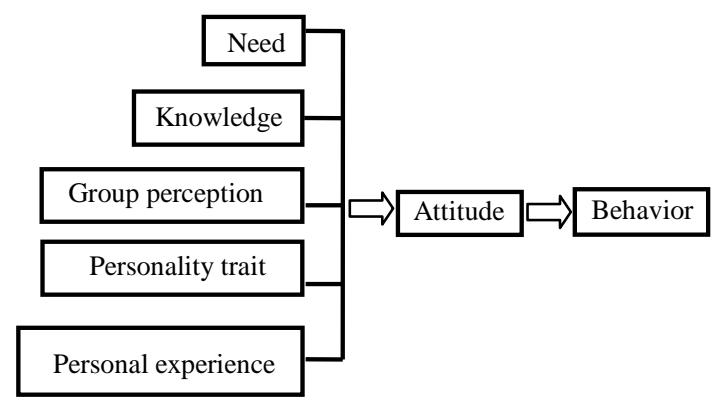

Figure 1. Factors impacting formation of attitude and relations between attitude and behavior.

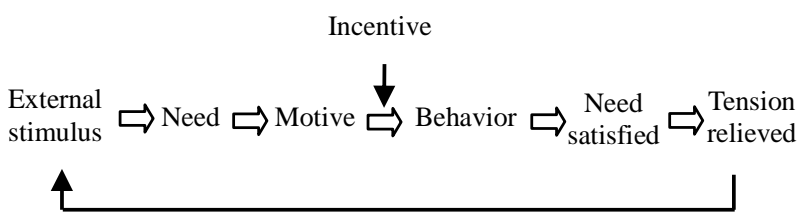

Figure 2. Process of incentive. 
will develop toward the expected direction and then reach the goal of risk control.

\section{Food Supply Chain Safety Risk Prevention and Control Based on Behavior}

The food supply chain safety risks exist in all aspects of the food chain. Although these risks occur in different links, what they have in common is that people's participation. In other words, a large part of the food supply chain safety risks can be attributed to human behavior. Therefore, there is a great point in controlling participants' behavior when preventing and controlling the food supply chain safety risk. Individual behavior is mainly affected by two factors, which are attitude and the motivation. The attitude of individual decides the direction of behavior, while external motivation affects performance of the individual behavior. Hence the prevention and control of food chain safety risk based on behavior should focus on these two aspects. Figure 3 demonstrates a mechanism of food supply chain safety risk prevention and control from a behavioral perspective. The upper part of Figure $\mathbf{3}$ is the integration of Figures $\mathbf{1}$ and 2. External stimulus, as the beginning of incentive process showed in Figure 2, can be separated into positive stimulus and negative one. According to incentive process, positive stimulus can induce safe behavior. As for the negative stimulus, it should be avoided so as to curb the unsafe behavior. Therefore the food supply chain safety risk is reduced by controlling human behavior.

\subsection{Correcting Food Supply Chain Participants' Attitudes}

Attitude is a tendency of acts. Correcting attitude plays a key role in human behavior. Wrong attitude will lead to unsafe behavior. Hence, participants' attitude should be

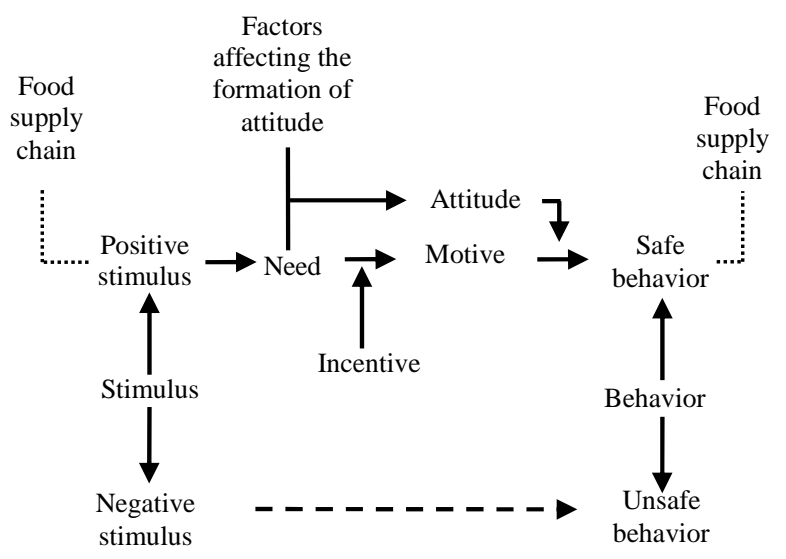

Figure 3. Preventing and controlling unsafe behavior in food supply chain. corrected at first in order to avoid unsafe behavior in food supply chain. When correcting their attitude, it's necessary to understand and control the factors that affect the formation of attitude. When those factors are under control, attitude can be corrected fundamentally, guiding to correct behavior. The main factors are need, knowledge, group perception, personality traits and individual experience.

Individual needs, personality traits, and personal experience is highly subjective and personalized, belonging to internal traits, while knowledge and group perception is more objective and interlinked, belonging to external environment. Accordingly, the latter two factors that influence the formation of attitude are easier to be controlled for their objectivity, which is of help to food supply chain safety risk prevention and control. In addition, personal trait and subjective characteristics can be changed correspondingly through external environment impact like education, training or group perception. According to Lewin field theory, human behavior is a function of external environment and personality, which indicates that external environment is one of critical elements that impact human behavior. Therefore, knowledge and external environment are two critical points of correcting food supply chain participants' attitudes as well as essential factors that influence the formation of attitude.

\subsubsection{Education and Training}

The information and knowledge individuals obtain in working or studying influence the formation of attitude. They tend to compare the knowledge they recently receive to their existing attitude, and adapt new knowledge into existing attitude or change former cognition system in order to develop new attitude through that comparison. Some food supply chain participants are short of relevant knowledge or perform incorrect operations, which results in safety issues. Take China's food supply chain for example, food and drink industry in China is one of main employers that attract lots of laid-off workers and rural surplus labor source who are lacking health knowledge and operation skills, which adds underlying safety troubles to this industry. By education and training, publicity of health knowledge or practice of safe operation, employed personnel could get their safety perception improved, attitude corrected, behavior restrained, therefore food safety risks based on behaviors get lowered through restraining behavior. D. R. Johnson (1995) [15] analyzed the behavior-oriented food safety training received by a group of objects, finding that after people attended food safety behaviors training which emphasized on correct habitual behaviors, these training behaviors had been reinforced and put into practice, and finally developed into habits. 
As a consequence, in preventing and controlling food supply chain safety risk, food industry associations ought to play an active role in industry related training. For example, in food supplying link, the upstream industry is usually composed of agriculture, forestry, animal husbandry and aquaculture industry, in which the potential safety hazard hidden in breeding and cultivation process is inevitably passed to downstream food supply chain. Consequently, employees in upstream industry should receive regular related education and training in terms of pesticide and agriculture chemicals usage, poultry feed and the like. Through long-term education and training, food suppliers incline to change their knowledge system and develop new attitude by comparing newly received knowledge to the former. Education and training can make the food suppliers develop habitual safe behavior in daily operation, thereby reducing the possibility of food supply chain safety risk caused by unsafe behaviors.

\subsubsection{Utilizing the Influence of Group Cognition}

The attitude of individual is usually affected by his surrounding group, such as family, school, community he belongs to and the like. This is because individual's sense of group identity makes them more likely to accept the norms of group or receive similar education or else. In China, food safety incidents have happened frequently in recent years, which have much to do with the status quo of food processing industry. Food processing Industry consists of a great number of enterprises of small size, lacking of technology and connection between each other. This low diversity of enterprises makes it hard to strengthen industry scale and to form a standardized mechanism, which inevitably misleads some individual organizations to adopt improper behavior, resulting in adding to food supply chain safety risk.

Therefore, integration of food processing industry is a necessary measure to be taken. Government and industry associations should guide to build up industry cluster, which helps facilitate collaboration and encourage learning among enterprises, improving the managerial efficiency and effectiveness within the food industry. Meanwhile, related laws and regulations need to be implemented rigorously. The rigor of laws and regulations can keep in check human behaviors in the food processing. Thus, each enterprise exposes itself to a scaled and standardized management mechanism, influenced by other organizations among the cluster. Through frequent communication and cooperation among enterprises, the perception of safe behavior of each organization gradually converge to form an industry standard, in order to curb unsafe behaviors of individual enterprise.

\subsection{Providing Incentives}

For food supply chain with a high complexity, motiva- tion is a critical factor to improve the quality of food supply chain operation, so as to lower food supply chain safety risk. S. A. Cohen et al. (2007) [16] carried out a survey that demonstrated incentives were much more likely put into use in supply chain with higher complexity. Sometimes that food supply chain participants behave unsafely is not because of incorrect attitude, but the lack of motivation, which undermines the driving power to behave safely. According to Lewin field theory, human behavior tends to be influenced by surrounding environment. When unsafe behavior in external environment dominates, individuals incline to lose drive of developing safe behavior. However, if performing safe acts are rewarded, then the rewards become an incentive, prompting a need for safe behavior. The higher the availability of rewards is, the more likely individuals willingly to behave safely, so as to avoid unsafe behavior.

Correct attitude is not simply enough to develop safe behavior, motivation is another drive. According to motivation theory, positive stimulus which breeds need for safe acts is necessary in preventing unsafe behavior, and so is the inducement that makes the actors feel that safe behavior can satisfy their needs. On the other hand, negative stimulus should be restrained, avoiding behaviors giving rise to problems. Positive stimulus is rewarding those behaviors guaranteeing and improving food safety, therefore these safe behaviors get reinforced and can be adopted repeatedly. On the contrary, negative stimulus is to punish those behaviors triggering food supply chain safety risk, and these unsafe behavior get restrained, so as to lower risk.

\subsubsection{Behavior Reinforcement}

Reinforcement is a psychological term, which means the connection between the increase of some stimulus and the organism. Behavior reinforcement is the process of increasing, decreasing or eliminating some behavior through consistently changing of environmental stimulus. Reinforcement can be divided into positive ones and negative ones. Both of them are processes of reinforcing behavior, the difference between them is the way of changing the environmental stimulus. As for positive reinforcement, behavior is reinforced through increasing stimulus followed by the behavior. On the contrary, negative reinforcement is through removing stimulus to strengthen the behavior.

In the process of management, most people use positive reinforcement to encourage the repetition of correct behavior, such as praise, promotion, payment of prizes and grant award. Thus the safe behavior in food supply chain can be increased by positive reinforcements. Heineken used to run a "Forecast accuracy incentive program", which aimed at encouraging the beer distributors to increase the quality of goods demand prediction. 
Goods demand prediction is an important way of increase the efficiency of supply chain and a key to decrease the incidence of supply safety risk. By rewarding American express, one of their distributors, gift cards, Heineken increase enthusiasm of the prediction workers substantially, thus the accuracy of monthly prediction from 2004 to 2005 increased 10\% [17]. Providing the food supply chain participants, whether they are individuals or enterprises, some incentives such as bonuses, subsidies and allowances, can increase the enthusiasm of food industry and its employees. It reinforces safe behaviors and lowers the risk of unsafe behaviors. In the use of positive reinforcement, the role of negative reinforcement should not be ignored. For the food production and processing enterprises, the tax is a large part of expense, which can be considered as a negative stimulus. Decreasing the tax benefits the enterprises and surely can be gladly accepted. Thus the negative stimulus of decreasing the tax will increase the behavior and act like an incentive to enterprises. Therefore, the use of tax-decrease can reinforce the safe behaviors of food production and processing enterprises and also lower the risk of food supply chain.

\subsubsection{Improving Availability of Incentives}

According to motivation theory, when the external environment stimulates an individual, the needs and motivation formed correspondingly is not enough to develop a behavior. In the process of behavior shaping, an inducement that makes individuals feel that their needs can be satisfied is required. That is to say, only higher incentive availability can make individuals adopted expected behaviors. Take the above discussion on positive reinforcement for an example, the rewards given to raw material supplier need to meet at least two points to be considered high availability: 1) The rewards should be much more lucrative than the profits obtained through adopting unsafe behaviors. If the satisfaction brought by the rewards is not sufficient to replace the profits gained from unsafe behavior, then suppliers will continue to adopt unsafe behavior; 2) The rewards need to be awarded in time. The rewards that are not honored punctually will undermine or even eliminate the effectiveness of motivation, because incentives will not work if there is no connection between safe behavior and reward due to time delay.

\section{Conclusion}

The food safety issue has become a hot topic at home and abroad. In recent years, the frequent domestic food safety incidents have made China's food export industry suffer from a crisis of confidence, especially the "food threat theory" concocted by the developed countries has seriously affected China's food exports. Food supply chain safety risks arise from the supply chain, the food supply chain safety risk management is one of the internationally recognized concepts of food safety management, and also is the basis of establishing food safety monitor measures [18]. Food safety is not only related to personal health and safety, but also to national security, and to the end, to the human welfare. The whole world should collaborate together, and establish a scientific and rational system of public food safety. From raw materials to processing, packaging, distribution and trade, and even on the table, food supply and food processing should strictly implement the relevant procedures and regulations. The effects of human behaviors on food supply chain ought to be strongly emphasized in preventing and controlling food supply chain safety risks, due to the food supply chain participants' behavior is closely related to the operation of the entire food supply chain. The two factors that influence human behavior are attitude and motivation, which are the entry points of controlling behavior. On the aspect of attitude, the occurrence of unsafe acts can be circumvented by arousing needs, knowledge training, applying the effects of group cognition, and the assimilation of the individuals. In motivation, the mutual cooperation of positive and negative reinforcement and incentive availability are two key factors. In this paper, the theoretical analysis of the factors of human behaviors in the prevention and control of food supply chain safety risk provides new ideas and methods for future research in related fields.

\section{REFERENCES}

[1] P. P. Wang, "Relationship between Food Supply Chain and Food Safety,” Meat Research, No. 1, 2010, pp. 59-64.

[2] R. C. Cui and X. P. Li, “The Reason of Farmers' Implicit Breach of Contract in Order Agriculture and Ways of Avoiding," Journal of Jining College, Vol. 33, No. 1, 2012, pp. 106-110.

[3] Z. G. Wang, T. G. Li and J. Peng, "The Analysis on Farmers' Usages of Chemicals under the Food Safety Regulation," Journal of China Agricultural University, Vol. 16, No. 3, 2011, pp. 164-168.

[4] Z. Z. Qiu and Y. Yang, "The Analysis on Consuming Behavior Based on Food Safety," Journal of Anhui Agricultural Science, Vol. 36, No. 8, 2008, pp. 3432-3433.

[5] L. Jiang, "Food Safety: Public Perception, Mood and Behavior," Journal of Jiangnan University (Humanities \& Social Sciences), Vol. 10, No. 6, 2011, pp. 89-93.

[6] C. M. Fan, J. M. Jia and H. Q. Li, "Research on Public Risk Perception and Reacting Behavior in Food Safety Incidents,” Public Management, Vol. 24, No. 1, 2012, pp. 163-176.

[7] Z. Y. Zhang and G. H. Yang, "The Game Analysis on Food Processing Safety and Government Surveillance,” Technology Information, No. 29, 2009, pp. 589-590.

[8] P. D. Liu and T. J. Wang, "Research on Risk Evaluation in Supply Chain Based on Grey Relational Method,” 
Journal of Computer, Vol. 3, No. 10, 2008, pp. 28-35.

[9] L. Manning, R. N. Baines and S. A. Chadd, "Ethical Modeling of the Food Supply Chain,” British Food Journal, Vol. 108, No. 5, 2006, pp. 358-370. doi:10.1108/00070700610661330

[10] B. Bigliardi and E. Bottani, "Performance Measurement in the Food Supply Chain: A Balanced Scorecard Approach,” Facilities, Vol. 28, No. 5-6, 2010, pp. 249-260. doi:10.1108/02632771011031493

[11] T. Tokar, "Behavioral Research in Logistics and Supply Chain Management,” International Journal of Logistics Management, Vol. 21, No. 1, 2010, pp. 89-103. doi:10.1108/09574091011042197

[12] Q. Mao and J. Ge, "Review of BOD Management Base on Behavioral Perspective,” Journal of Jinling Institute of Technology (Social Science), Vol. 24, No. 3, 2010, pp. 711.
[13] S. P. Robinson, "Essentials of Organizational Behavior," Electronic Industry Press, Beijing, 2005, p. 21.

[14] W. D. Huang, Y. Liu and Q. Xu, "Organizational Behavior,” Tsinghua University Press, Beijing, 2005, p. 43.

[15] D. R. Johnson, "Behavior-Based Food Safety Training Using Hazard Analysis Critical Control Point Principles," Ph.D. Thesis, Kansas State University, Manhattan, 1995.

[16] S. A. Cohen, S. Kulp and T. Randall, "Motivating Supply Chain Behavior: The Right Incentives Can Make All the Difference," Supply Chain Management Review, Vol. 11, No. 4, 2007, pp. 18-24.

[17] B. Dershem, "Financial Incentives Improve the Supply Chain: Heineken's Journey," The Journal of Business Forecasting, Vol. 26, No. 1, 2007, pp. 32-35.

[18] C. He, "Discussion on China’s Food Supply Chain Safety Risk Control Mechanism,” Academic Exchange, No. 11, 2011, pp. 75-78. 